\title{
Chandra Observation of the Pulsar Wind Nebula Powered by PSR J1135-6055
}

\author{
Xiying Zhang \\ Universitat de Barcelona/ICCUB \\ E-mail: xzhangeicc.ub.edu

\section{Pol Bordas} \\ Universitat de Barcelona/ICCUB \\ E-mail: pbordas a fqa .ub. edu
}

\begin{abstract}
The supersonic motion of a pulsar in the ambient medium usually renders pulsar wind nebulae with morphologically bow-shaped shocks and/or cometary tails. Chandra, with its unprecedented angular resolution and high sensitivity has proved a great success in the detection and precise characterisation of these fast-moving pulsars. In particular, with an increasing number of detected supersonic pulsar wind nebula systems, an unusual outflow structure misaligned with respect to the pulsar motion has been observed in several cases. Here, we report recent results on the analysis of the extended emission around PSR J1135-6033 and the pulsar itself based on archival Chandra data.
\end{abstract}

Keywords: Pulsar jets; Pulsar wind nebulae; X-rays; Fast-moving pulsars

High Energy Phenomena in Relativistic Outflows VII - HEPRO VII

9-12 July 2019

Facultat de Física, Universitat de Barcelona, Spain 


\section{Introduction}

X-ray observations of pulsar wind nebulae (PWN) obtained with Chandra have revealed a variety of nebular morphologies (see e.g.[1]). PWN produced by young, subsonically moving pulsars like the Crab tend to acquire torus-jet like PWN morphologies while old, supersonically moving pulsars and their nebula (SPWN) typically display bow-shock cometary-tail emission [2], the morphology of which provides detailed information on the proper motion of the pulsar and the properties of both the ambient medium and the pulsar shocked wind plasma. Furthermore, a new type of jet-like outflows has been observed in a few SPWN systems (see e.g. [3]), displaying extreme morphological properties. The study of these outflow structures may help in the understanding of the formation of pulsar jets, its ejection mechanism and their propagation through the medium.

The pulsar PSR J1135-6055 was discovered with the Fermi-LAT in a blind search as an energetic gamma-ray only pulsar [4]. The main properties of this system are provided in Table 1. The analysis of the archival Chandra data obtained from the observation of the supernova remnant (SNR) G293.8+0.6, covering also PSR J1135-6055, revealed the presence of the pulsar and its nebula in the X-ray band [5]. The SNR G293.8+0.6 was on the contrary not detected at these energies, despite that the two systems may be associated. The morphological properties of the extended emission around PSR J1135-6055 obtained with these Chandra observations unveiled a fast-moving system, displaying also large-scale jet-like structures at both sides of the pulsar [5]. A map of the region containing both SNR G293.8+0.6 and PSR J1135-6055 is shown in Fig. 1, a radio image of the supernova remnant taken at $843 \mathrm{MHz}$ [6]. The hugely asymmetric morphological shape of this SPWN has been considered as a prototype of a mixed "Frisbee-Rifle Bullet" configuration by simulating and reproducing the key features of bow-shock PWNe [7]. In this article we report on the analysis of all the archival Chandra data sets covering the source, focusing on the morphological properties of this SPWN to constrain from observations the nature of its jet-like outflows.

\begin{tabular}{ccccc}
\hline $\begin{array}{c}d \\
\mathrm{kpc}\end{array}$ & $\begin{array}{c}\log \dot{E} \\
\mathrm{erg} \mathrm{s}^{-1}\end{array}$ & $\begin{array}{c}\log \tau \\
\mathrm{yrs}\end{array}$ & $\begin{array}{c}B_{11} \\
10^{11} \mathrm{G}\end{array}$ & $\begin{array}{c}v_{\perp} \\
\mathrm{km} \mathrm{s}^{-1}\end{array}$ \\
\hline$\sim 2.8$ & 36.23 & 4.36 & 30.5 & $<330$ \\
\hline
\end{tabular}

Table 1: Parameters of PSR J1135-6055 (from [2]). 




Figure 1: Radio image of the supernova remnant G293.80+0.6 taken by MOST at $843 \mathrm{MHz}$ [6]. The white cross shows the position of PSR J1135-6055.

\section{Observations, Data Analysis and Results}

Besides the initial Chandra observation aiming at SNR G293.8+0.6 which covered and revealed the presence of the pulsar PSR J1135-6055 and its wind nebula in X-rays, there are two more archival Chandra observations performed afterwards focusing on the SPWN system itself. A list of the Chandra observations that we make use of is given in Table 2.

\begin{tabular}{cccc}
\hline Observation ID & Date & Exposure $(\mathrm{ks})$ & Instrument \\
\hline 3924 & $2003-08-24$ & 36.11 & ACIS-S \\
15966 & $2014-12-30$ & 55.54 & ACIS-S \\
17572 & $2015-01-03$ & 35.73 & ACIS-S \\
\hline
\end{tabular}

Table 2: Publicly available Chandra observations covering PSR J1135-6055.

Data-sets corresponding to all observations were performed in Chandra's VERY FAINT timed exposure mode by using the instrument's ACIS-S detector. The position of PSR J1135-6055 is in all cases on top of the Chandra back-illuminated ACIS S3 chip. Data processing was performed using 
the Chandra Interactive Analysis of Observations (CIAO) software [8], version 4.11, together with the publicly available Chandra Calibration Database (CALDB) in version 4.8.2. All observations were reprocessed using chandra_repro in VFAINT background cleaning mode. Unless otherwise noted, we consider only events on S3 (ccd_id=7) chip and restrict the energy range to 0.5 $7.0 \mathrm{keV}$.

We used the fluximage CIAO tool to generate both the exposure map and the broad-band exposure-corrected flux image for each observation data-set. We then employed the Voronoi Tessellation and Percolation source detection tool vtpdetect on the reprocessed level=2 event file using the previously generated exposure map, as this method is optimized for the detection of faint, low surface-brightness extended sources [9]. The generated source lists were used to remove sources in the field of view (FoV) in order to get source-free background datasets. We then checked the background data for the presence of X-ray flares by extracting and filtering the background light-curve (in temporal bins of $200 \mathrm{~s}$ width) for deviations larger than 3 sigma. Background $\mathrm{X}$-ray flares were found and subsequently removed only in the first two observations. The effective final exposures that we got for each observation are $32.36 \mathrm{ks}, 55.14 \mathrm{ks}$, and $35.73 \mathrm{ks}$, summing up a total of $123.23 \mathrm{ks}$.

In order to merge all the data to perform a morphological analysis of PSR J1135-6055 and its surroundings, the WCS reference system of observation ObsID 15966 was used as reference since it is the longest of the two later consecutive observations and we assume that between these two there is no significant difference in the astrometry. Nevertheless, to correct for possible small discrepancies in the astrometry of the corresponding event files, we applied the wavdetect tool, based on a Mexican-hat wavelet source detection method [10], to detect point sources in both Chandra observations: ObsID 3924 and ObsID 15966. The two output source lists were then cross-matched to re-project the aspect solution file of ObsID 3924 using reproject_aspect. By running the CIAO tools wCS_match and wCS_update we ensured a common WCS reference system for all observation data-sets.

The merging of the three observations was performed using the CIAO routine merge_obs, with binsize set to 1 . Merged event files and exposure-map corrected flux images were obtained for broad-band energy range $(0.5-7.0 \mathrm{keV})$, as well as three separate intervals: soft $(0.5-1.2 \mathrm{keV})$, medium (1.2-2.0 keV), and hard (2.0-7.0 keV). For each energy range, a map of the retrieved raw counts was divided by the exposure map corresponding to that band, and then normalised by the exposure time to produce the final flux image reported in Fig. 2, which displays the broad energy band (0.5-7 keV) exposure-corrected flux image around PSR J1135-6055. From this map, in addition to the SPWN itself, two asymmetric jet-like structures emanating from the SPWN can be clearly distinguished. The western jet displays an "arc" shape, probably related to the pressure exerted by the surrounding medium. The eastern jet-like feature, the more diffused one, instead, displays a remarkable rectilinear structure. When observed separately at low, medium and high energies, the two jet-like structures appear to be mainly emitting in the latter bands, with little contribution at low-energies (see Fig. 2).

Surface brightness profiles were extracted for both the eastern and western jet-like features at increasing distances from the PSR location. Whereas the eastern jet (left panel in Fig. 3) displays a rather smooth flux profile, the western feature displays a moderately significant enhancement about 

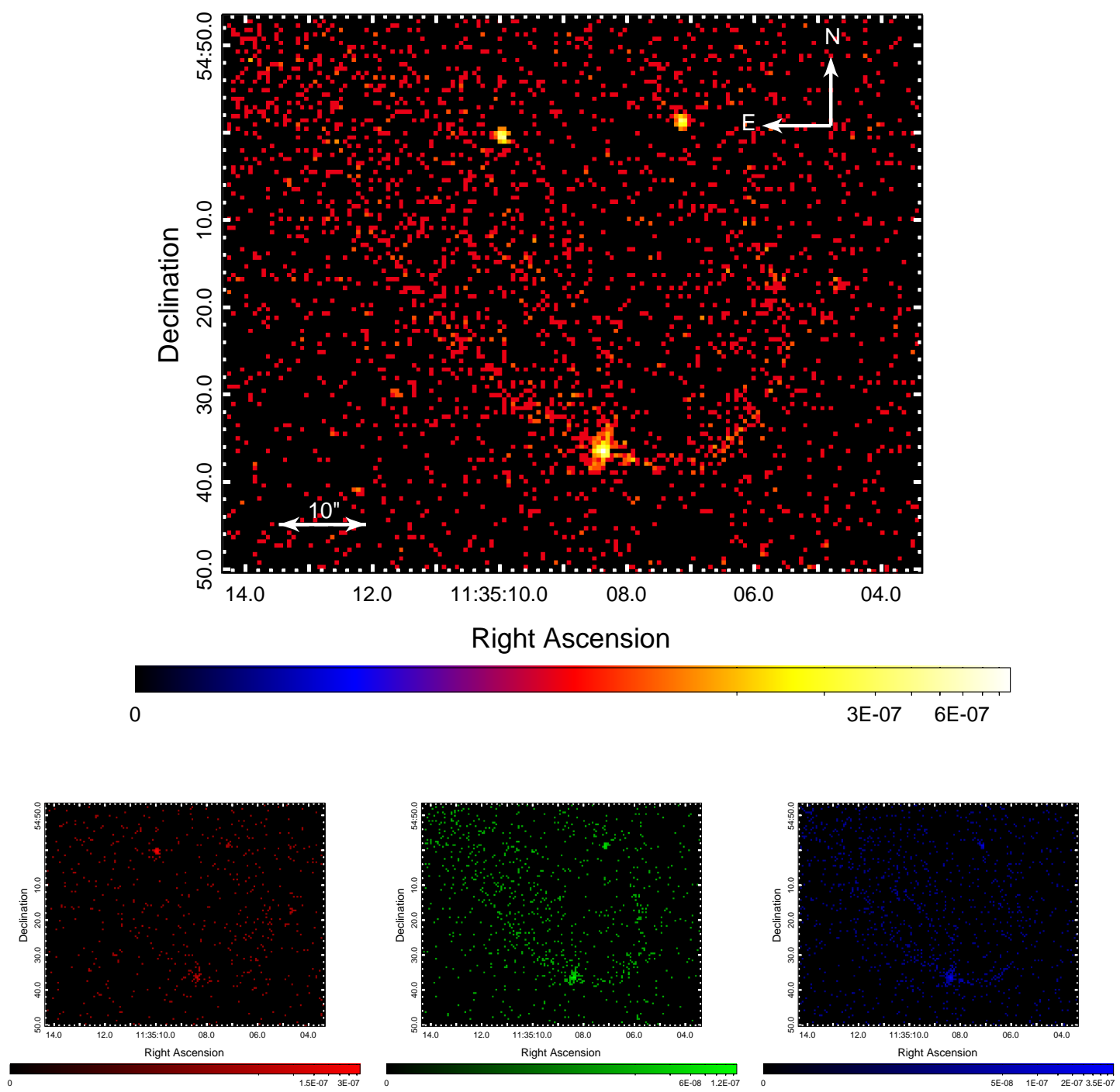

Figure 2: Exposure-corrected flux maps of the extended emission around PSR J1135-6055 for energies in the range 0.5-7.0 keV (upper panel), $0.5-1.2 \mathrm{keV}$ (bottom-left panel), 1.2-2.0 keV (bottommiddle panel), and 2.0-7.0 keV (bottom-right panel).

18 arc-secs away from the PSR location, followed by a decrease, and recovering afterwards to a relatively smooth profile. These profiles further highlight the asymmetry of the two jet-like structures. An extended profile has also been extracted for PSRJ 1135-6055 and its immediate surroundings using 8 rectangular slices along the north-west direction (see Fig. 4). The SPWN displays a clear extended emission along this axis, with a moderately broad flux profile which extends much beyond Chandra's point spread function (PSF). This extended emission is interpreted as the nebula powered by the central pulsar. 

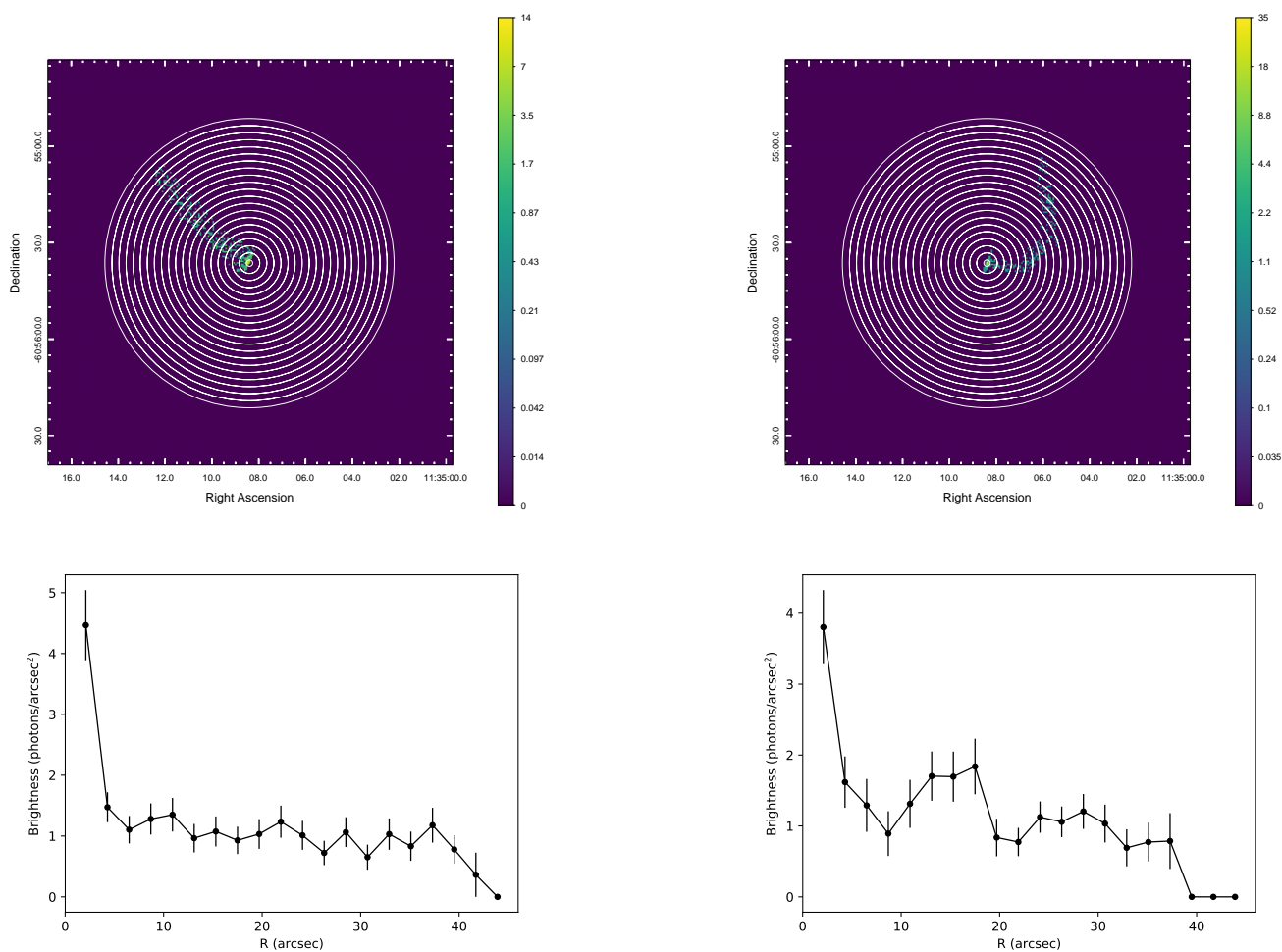

Figure 3: Upper panels: extraction schemes for the eastern (left) and western (right) jet-like structures around PSR J1135-6055. Bottom panels: surface brightness profiles along the eastern (left) and western (right) jets.

\section{Discussion}

The morphological analysis of PSR J1135-6055 reported in Sect. 2 has revealed the presence of extended nebular emission around PSR J1135-6055 together with two asymmetric jet-like features protruding from the pulsar location. The angular size of these jets is $\sim 30 "-40 "$; and for an estimated distance to the source of $\sim 2.8 \mathrm{kpc}$ ([11]), this translates into a jet length of at least $l_{\text {jet }} \sim$ $1.2-1.6 \times 10^{18} \mathrm{~cm}$. Note that this estimate assumes only the projection on the plane of the sky. If jets are propagating at a direction which subtends an angle $\theta$ with respect to this plane, their length will increase by a factor $(\cos \theta)^{-1}$. As for the nebular emission around PSR J1135-6055, an extension at the level of about 2"-3" has been retrieved for the "tails" at both sides of the PSR (see Fig. 4). At $2.8 \mathrm{kpc}$, this translates into a physical extension of $2 \times 10^{17} \mathrm{~cm}$.

The origin of the nebular emission and the two prominent jet-like features around PSR J11356055 is uncertain. Although the central extended source and these jet-like features could in principle be unassociated, the low probability of the coincidence for both jets' alignment right towards the pulsar position strongly argues against it. A preliminary spectral analysis of both the SPWN and the two jet components (not reported here) provides also with similar values for the hydrogen column density $N_{\mathrm{H}}$, further strengthening their association. Furthermore, similar morphologies have been previously observed in other well-studied SPWN, see e.g. [2]. 
Assuming that PSR J1135-6055 is moving supersonically through the surrounding interstellar medium (ISM), the morphology of its SPWN is expected to display a bullet-like morphology, with extended emission trailing the motion of the compact source. The radio map reported in Fig. 1 displays the diffuse radio emission produced by the SNR G293.8+0.6, including a bright nebular emission centred around $\mathrm{RA}=11: 34: 50, \mathrm{DEC}=-60: 53: 00$. This latter nebular emission is elongated along a direction which roughly coincides with the extended X-ray emission around PSR J11356055 (see Fig. 4). A possible interpretation could account for the association between these two observables: freshly accelerated electrons emitting synchrotron X-rays in the relatively compact PWN trailing the pulsar motion may cool down and radiate synchrotron radio emission at larger distances in a much more expanded nebular structure (see e.g. a similar pattern observed in the Lighthouse Nebula [12])

Extended emission ahead of the central compact object could pinpoint the location of the termination shock, $R_{\mathrm{TS}}$, formed when the pressure of the pulsar wind is balanced by the inertia of the swept-up ambient medium. The distance between the pulsar and the head of the termination shock can be expressed through [13]:

$$
R_{\mathrm{TS}}=\left(\dot{E} / 4 \pi c \rho_{\mathrm{ISM}} v_{\mathrm{PSR}}^{2}\right)^{1 / 2}
$$

where $\dot{E}$ is the pulsar spin-down power, $\rho_{\text {ISM }}$ is the ambient medium density and $v_{\text {PSR }}$ is the pulsar velocity. Setting $R_{\mathrm{TS}}=2 \times 10^{17} \mathrm{~cm}, \dot{E}=10^{36} \mathrm{erg} \mathrm{s}^{-1}$ [2] and assuming $\rho_{\mathrm{ISM}} \approx 0.1-1.0 \mathrm{~m}_{\mathrm{p} \mathrm{cm}} \mathrm{cm}^{-3}$, this translates into a pulsar velocity of $v_{\mathrm{PSR}} \sim 125-390 \mathrm{~km} \mathrm{~s}^{-1}$.

The jet-like features, if these are true ballistic jets propagating at a given angle with respect to the supersonic motion direction, should display signatures of bending due to the ram pressure exerted by the surrounding ISM. In this regard, the western jet-like feature reported above (see Figs. 2 and 3) does indeed display a curvature which could well be associated to such bending effect. On the contrary, the fact that the eastern jet does not show similar bending features is somehow intriguing.

Quasi-rectilinear X-ray jet-like features have been detected in the last years in a few SPWNs ([2, $14,12,15,16])$. In these cases, the outflows are largely misaligned with respect to the pulsar proper motion (in contrast with slow-motion pulsars like the Crab and Vela, where jets propagate along the proper motion direction). Furthermore, in a number of cases these jet-like structures show no signature of bending, despite that the powering pulsar has proper motion velocities of several $\times 100 \mathrm{~km} \mathrm{~s}^{-1}$. This prompted for an alternative interpretation for these outflows, based on the idea that high-energy particles can escape the bow-shock nebula around the pulsar and diffuse into ISM magnetic field lines [17]. In this scenario, the rectilinear geometry of these jet-like features is just due to the intrinsic planar-parallel structure of the underlying ISM magnetic field. Recent numerical simulations seem to confirm such scenario, predicting as well possible asymmetries in the "jet" and "counter-jet" emission [7].

The jets reported here could also be interpreted in such diffusion framework, particularly the eastern outflow, which displays no signature of bending. In order to distinguish between this and more classical "ballistic" jet scenarios, spectral studies are needed to constrain the magnetic field within the nebula as well as the characteristic energy of the emitting leptons. Spectral features may also 

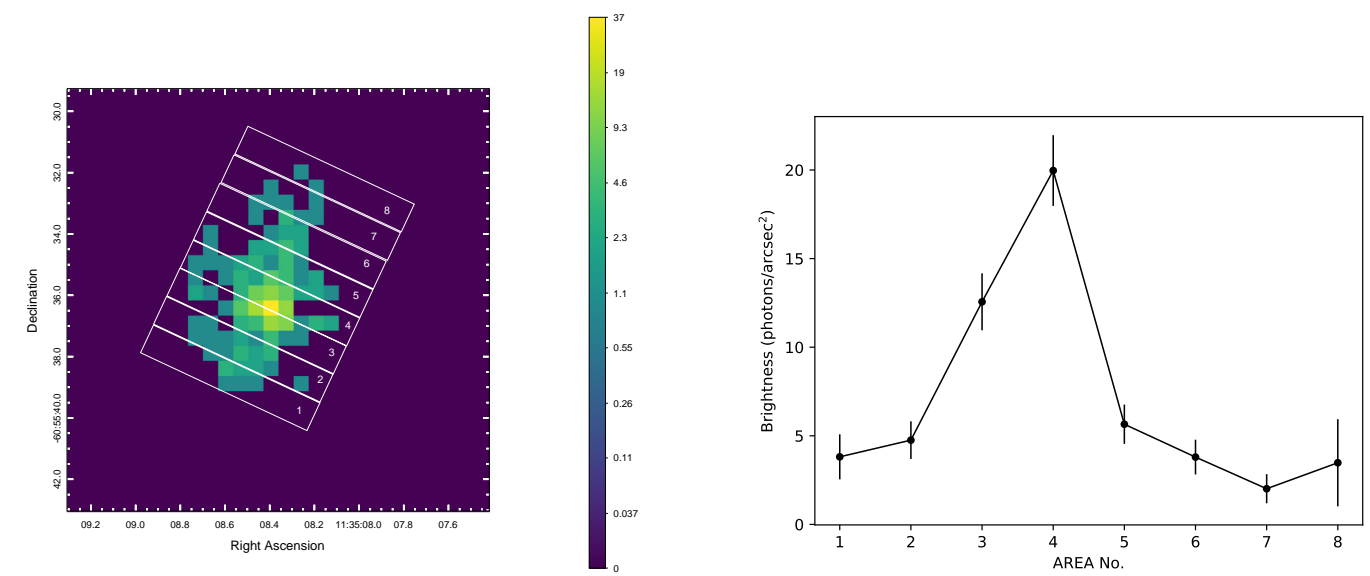

Figure 4: Exposure-corrected flux maps of the central part of the SPWN (left panel), together with the extraction scheme (grid with eight 1"- width slices) used to produce the brightness profile around PSRJ1135-6055 (right panel).

help to individuate breaks due to radiation cooling favouring one or another scenarios. These studies are in preparation for a forthcoming paper.

\section{Conclusions}

A preliminary Chandra archival data analysis on the fast-moving pulsar PSR J1135-6055 has revealed the presence of extended nebular emission around the source together with large-scale jet-like features which resemble the ones observed in similar systems, e.g. the Guitar Nebula, the Lighthouse nebula, the PWN of PSR J1509-5058 and the Mushroom PWN around PSR B0355+54. These jet-like features seem to emit mainly at high energy X-rays, with little emission at lower energies. The eastern jet in PSR J1135-6055 displays no signatures of bending due to the ram pressure exerted by the surrounding medium, despite the relatively high proper motion velocity derived for the pulsar $\gtrsim 125 \mathrm{~km} \mathrm{~s}^{-1}$. The origin of these "jets" is therefore unclear, with different interpretations based either on a ballistic jets scenario or diffusion into the ambient magnetic field lines. A more detailed spectral analysis of PSR J1135-6055 's jet-like structures is in preparation, which will allow to better evaluate the nature of these outflows.

ACKNOWLEDGEMENTS: Xiying Zhang (XZ) and Pol Bordas (PB) are grateful to J. M. Paredes and V. Bosch-Ramon for the fruitful scientific discussions related to this research project. $\mathrm{XZ}$ and $\mathrm{PB}$ acknowledge the support by the Institut de Ciències del Cosmos (ICCUB) as well as the excellent working conditions at the Departament de Física quàntica i Astrofísica at the Universitat de Barcelona. PB acknowledges the financial support from the Generalitat de Catalunya through a Beatriu de Pinós grant.

\section{References}

[1] O. Kargaltsev and G. G. Pavlov, Pulsar Wind Nebulae in the Chandra Era, in 40 Years of Pulsars: Millisecond Pulsars, Magnetars and More, C. Bassa, Z. Wang, A. Cumming and V. M. Kaspi, eds., vol. 983 of American Institute of Physics Conference Series, pp. 171-185, Feb, 2008, 0801.2602 , DOI. 
[2] O. Kargaltsev, G. G. Pavlov, N. Klingler and B. Rangelov, Pulsar wind nebulae created by fast-moving pulsars, Journal of Plasma Physics 83 (2017) 635830501 [1708. 004 56].

[3] L. Pavan, G. Pühlhofer, P. Bordas, M. Audard, M. Balbo, E. Bozzo et al., Closer view of the IGR J11014-6103 outflows, A\&A 591 (2016) A91 [1511.01944].

[4] A. de Luca, M. Marelli and P. A. Caraveo, X-Ray observations of "gamma-ray only" PSRs, in The 2011 Fermi Symposium, The 2011 Fermi Symposium, May, 2011.

[5] M. Marelli, The X-ray behaviour of Fermi/LAT pulsars, arXiv e-prints (2012) arXiv:1205.1748 [1205.1748].

[6] C. M. Olbert, J. W. Keohane and E. V. Gotthelf, A Soft X-ray Point Source in G293.8+0.6, in American Astronomical Society Meeting Abstracts, vol. 203 of American Astronomical Society Meeting Abstracts, p. 39.07, Dec, 2003.

[7] M. V. Barkov, M. Lyutikov, N. Klingler and P. Bordas, Kinetic 'jets' from fast-moving pulsars, MNRAS 485 (2019) 2041 [1804.07341].

[8] A. Fruscione, J. C. McDowell, G. E. Allen, N. S. Brickhouse, D. J. Burke, J. E. Davis et al., CIAO: Chandra's data analysis system, vol. 6270 of Society of Photo-Optical Instrumentation Engineers (SPIE) Conference Series, p. 62701V. 2006. 10.1117/12.671760.

[9] H. Ebeling and G. Wiedenmann, Detecting structure in two dimensions combining Voronoi tessellation and percolation, PRE 47 (1993) 704.

[10] P. E. Freeman, V. Kashyap, R. Rosner and D. Q. Lamb, A Wavelet-Based Algorithm for the Spatial Analysis of Poisson Data, ApJs 138 (2002) 185 [astro-ph/ 0108429 ].

[11] P. M. Saz Parkinson, M. Dormody, M. Ziegler, P. S. Ray, A. A. Abdo, J. Ballet et al., Eight gamma-ray Pulsars Discovered in Blind Frequency Searches of Fermi LAT Data, ApJ 725 (2010) 571 [1006.2134].

[12] L. Pavan, P. Bordas, G. Pühlhofer, M. D. Filipović, A. De Horta, A. O’Brien et al., The long helical jet of the Lighthouse nebula, IGR J11014-6103, A\&A 562 (2014) A122 [1309. 6792].

[13] J. M. Cordes, Pulsar Wind Nebulae, vol. 105 of Astronomical Society of the Pacific Conference Series, p. 393. 1996.

[14] S. P. Johnson and Q. D. Wang, The pulsar B2224+65 and its jets: a two epoch X-ray analysis, MNRAS 408 (2010) 1216 [1003.1724].

[15] N. Klingler, O. Kargaltsev, B. Rangelov, G. G. Pavlov, B. Posselt and C. Y. Ng, Chandra Observations of Outflows from PSR J1509-5850, ApJ 828 (2016a) 70 [1601. 07174].

[16] N. Klingler, B. Rangelov, O. Kargaltsev, G. G. Pavlov, R. W. Romani, B. Posselt et al., Deep Chandra Observations of the Pulsar Wind Nebula Created by PSR B0355+54, ApJ 833 (2016b) 253 [1610.06167].

[17] R. Bandiera, On the X-ray feature associated with the Guitar nebula, A\&A 490 (2008) L3 [0809.2159]. 